\title{
A Note on the Exponential G-Martingale
}

\author{
Yunsheng $L u^{1,2}$ and Yingying $\mathrm{Liu}^{2}$ \\ ${ }^{1}$ Glorious Sun School of Business and Management, Donghua University, 1882 West Yanan Road, Shanghai 200051, China \\ ${ }^{2}$ Department of Mathematics, Donghua University, 2999 North Renmin Road, Songjiang, Shanghai 201620, China
}

Correspondence should be addressed to Yunsheng Lu; luyunsheng@dhu.edu.cn

Received 14 November 2013; Accepted 26 November 2013

Academic Editor: Litan Yan

Copyright (C) 2013 Y. Lu and Y. Liu. This is an open access article distributed under the Creative Commons Attribution License, which permits unrestricted use, distribution, and reproduction in any medium, provided the original work is properly cited.

We get the exponential $G$-martingale theorem with the Kazamaki condition and tell a distinct difference between the Kazamaki's and Novikov's criteria with an example.

\section{Introduction and Main Result}

Motivated by various types of uncertainty and financial problems, Peng [1] has introduced a new notion of nonlinear expectation, the so-called $G$-expectation (see also Peng [2]), which is associated with the following nonlinear heat equation:

$$
\begin{gathered}
\frac{\partial}{\partial t} u(t, x)=G(\Delta u), \quad(t, x) \in[0,+\infty) \times \mathbb{R}, \\
u(0, x)=\varphi(x),
\end{gathered}
$$

where $\Delta$ is Laplacian and the sublinear function $G$ is defined by

$$
G(\alpha)=\frac{1}{2}\left(\bar{\sigma}^{2} \alpha^{+}-\underline{\sigma}^{2} \alpha^{-}\right), \quad \alpha \in \mathbb{R},
$$

with two given constants $0<\underline{\sigma}<\bar{\sigma}$. Together with the notion of $G$-expectations, Peng also introduced the related G-normal distribution, the G-Brownian motion, and related stochastic calculus under G-expectation, and moreover an Itô's formula for the $G$-Brownian motion was established. $G$-Brownian motion has a very rich and interesting new structure which nontrivially generalizes the classical one. Briefly speaking, a $G$-Brownian motion $B$ is a continuous process with independent stationary increments $B_{t+s}-B_{t}$ being $G$-normally distributed under a given sublinear expectation $\widehat{\mathbb{E}}$. A very interesting new phenomenon of $G$-Brownian motion $B$ is that its quadratic process $\langle B\rangle$ is a continuous process with independent and stationary increments, but not a deterministic process.
Recently, Xu et al. [3] got an exponential martingale theorem under $G$-framework with an assumption of Novikov's type. In this note, we will introduce the sublinear version of the classical Kazamaki condition. The main objective is to explain and prove the following theorem.

Theorem 1. If there exists an $\varepsilon_{0}>0$ such that

$$
\widehat{\mathbb{E}}\left[\exp \left\{\left(\frac{1}{2}+\varepsilon_{0}\right) \int_{0}^{T} H(s, \omega) d B_{s}\right\}\right]<\infty,
$$

then

$$
\mathscr{E}\left(B_{t}\right):=\exp \left\{\int_{0}^{t} H(s, \omega) d B_{s}-\frac{1}{2} \int_{0}^{t} H^{2}(s, \omega) d\langle B\rangle_{s}\right\}
$$

is a symmetric martingale under $\widehat{\mathbb{E}}$.

Under the classical case, the result is called Kazamaki's condition, and it can be recalled as follows, for any classical continuous martingale $M$, if

$$
E \exp \left(\frac{1}{2} M_{\infty}\right)<\infty
$$

then the martingale

$$
\exp \left\{M_{t}-\frac{1}{2}\langle M\rangle_{t}\right\}, \quad 0 \leq t \leq \infty,
$$

is uniformly integrable. Clearly, the Kazamaki principle is weaker than the Novikov condition. 
This note is organized as follows. In Section 2, we present some standard concepts and notations about G-Brownian motion and $G$-Expectation. In Section 3 we prove the above theorem and discuss some examples.

\section{Preliminaries}

In this section, we recall some concepts under the $G$-framework which are needed in our analysis. For more details, one can see Peng [1].

Let $\Omega \neq \emptyset$ be a given set and let $\mathscr{H}$ be a linear space of real valued functions defined on $\Omega$ such that $1 \in \mathscr{H}$ and $|X| \in \mathscr{H}$ for all $X \in \mathscr{H}$.

Definition 2. A sublinear expectation $\widehat{\mathbb{E}}$ on $\mathscr{H}$ is a functional with the following properties, for all $X, Y \in \mathscr{H}$, one has

(i) monotonicity: if $X \geq Y$, then $\widehat{\mathbb{E}}[X] \geq \widehat{\mathbb{E}}[Y]$;

(ii) constant preserving: $\widehat{\mathbb{E}}[c]=c$, for all $c \in \mathbb{R}$;

(iii) subadditivity: $\widehat{\mathbb{E}}[X]-\widehat{\mathbb{E}}[Y] \leq \widehat{\mathbb{E}}[X-Y]$;

(iv) positive homogeneity: $\widehat{\mathbb{E}}[\lambda X]=\lambda \widehat{\mathbb{E}}[X]$, for all $\lambda \geq 0$.

The triple $(\Omega, \mathscr{H}, \widehat{\mathbb{E}})$ is called a sublinear expectation space, and $\mathscr{H}$ is considered as the space of random variables on $\Omega$.

It is important to note that one can suppose that

$$
\varphi\left(X_{1}, \ldots, X_{d}\right) \in \mathscr{H}
$$

if $X_{i} \in \mathscr{H}, i=1, \ldots, d$, for all $\varphi \in C_{b, \mathrm{Lip}}\left(\mathbb{R}^{d}\right)$, where $C_{b \text {,Lip }}\left(\mathbb{R}^{d}\right)$ denotes the space of all bounded and Lipschitz functions on $\mathbb{R}^{d}$. In a sublinear expectation space $(\Omega, \mathscr{H}, \widehat{\mathbb{E}})$, a random vector $Y=\left(Y_{1}, \ldots, Y_{n}\right), Y_{i} \in \mathscr{H}$, is said to be independent under $\widehat{\mathbb{E}}$ from another random vector $X=$ $\left(X_{1}, \ldots, X_{m}\right), X_{i} \in \mathscr{H}$, if for each test function $\varphi \in$ $C_{b, \text { Lip }}\left(\mathbb{R}^{m+n}\right)$, one has

$$
\widehat{\mathbb{E}}[\varphi(X, Y)]=\widehat{\mathbb{E}}\left[\widehat{\mathbb{E}}[\varphi(x, Y)]_{x=X}\right] .
$$

Two $n$-dimensional random vectors $X$ and $Y$ defined, respectively, in the sublinear expectation spaces $\left(\Omega_{1}, \mathscr{H}_{1}, \widehat{\mathbb{E}}_{1}\right)$ and $\left(\Omega_{2}, \mathscr{H}_{2}, \widehat{\mathbb{E}}_{2}\right)$ are called identically distributed, denoted by $X \sim Y$, if

$$
\widehat{\mathbb{E}}_{1}[\varphi(X)]=\widehat{\mathbb{E}}_{2}[\varphi(Y)],
$$

for all $\varphi \in C_{b, \mathrm{Lip}}\left(\mathbb{R}^{n}\right)$.

Let $\underline{\sigma}$ and $\bar{\sigma}$ be two real numbers with $0<\underline{\sigma}<\bar{\sigma}$. A random variable $\xi$ in a sublinear expectation space $(\Omega, \mathscr{H}, \widehat{\mathbb{E}})$ is called $G$-normal distributed, denoted by $\xi \sim N\left(0,\left[\underline{\sigma}^{2}, \bar{\sigma}^{2}\right]\right)$, if for each $\varphi \in C_{b \text {,Lip }}(\mathbb{R})$, the function defined by

$$
u(t, x):=\widehat{\mathbb{E}}[\varphi(x+\sqrt{t} \xi)], \quad(t, x) \in[0, \infty) \times \mathbb{R},
$$

is the unique viscosity solution of the following nonlinear heat equation:

$$
\begin{gathered}
\frac{\partial}{\partial t} u(t, x)=G(\Delta u), \quad(t, x) \in[0,+\infty) \times \mathbb{R}, \\
u(0, x)=\varphi(x),
\end{gathered}
$$

where $\Delta$ is Laplacian and the sublinear function $G$ is defined by

$$
G(\alpha)=\frac{1}{2}\left(\bar{\sigma}^{2} \alpha^{+}-\underline{\sigma}^{2} \alpha^{-}\right), \quad \alpha \in \mathbb{R} .
$$

Example 3 (Peng [2]). Let $\xi \sim N\left(0,\left[\underline{\sigma}^{2}, \bar{\sigma}^{2}\right]\right)$. We then have

$$
\widehat{\mathbb{E}}[\varphi(\xi)]=\frac{1}{\sqrt{2 \pi} \bar{\sigma}} \int_{\mathbb{R}} \varphi(x) e^{\left(-1 / 2 \bar{\sigma}^{2}\right) x^{2}} d x
$$

for all convex functions $\varphi$ and

$$
\widehat{\mathbb{E}}[\psi(\xi)]=\frac{1}{\sqrt{2 \pi} \underline{\sigma}} \int_{\mathbb{R}} \varphi(x) e^{\left(-1 / 2 \underline{\sigma}^{2}\right) x^{2}} d x
$$

for all concave functions $\psi$.

Let now $\Omega=C_{0}\left(\mathbb{R}^{+}\right)$be the space of all real valued continuous functions on $[0, \infty)$ with initial value 0 , equipped with the distance

$$
\rho\left(\omega^{1}, \omega^{2}\right)=\sum_{i=1}^{\infty} 2^{-i}\left[\left(\max _{t \in[0, i]}\left|\omega_{t}^{1}-\omega_{t}^{2}\right|\right) \wedge 1\right], \quad \omega^{1}, \omega^{2} \in \Omega .
$$

We denote by $\mathscr{B}(\Omega)$ the Borel-algebra on $\Omega$. We also denote, for each $t \in[0, \infty)$,

$$
\Omega_{t}=\left\{\omega_{\cdot \wedge t}, \omega \in \Omega\right\}
$$

and $\mathscr{F}_{t}=\mathscr{B}\left(\Omega_{t}\right)$, where $x \wedge y=\min \{x, y\}$. We also denote the following:

(i) $L^{0}(\Omega)$ : the space of all $\mathscr{B}(\Omega)$-measurable real valued functions on $\Omega$;

(ii) $L^{0}\left(\Omega_{t}\right)$ : the space of all $\mathscr{B}\left(\Omega_{t}\right)$-measurable real valued functions on $\Omega_{t}$;

(iii) $L_{b}(\Omega)$ : the space of all bounded elements in $L^{0}(\Omega)$;

(iv) $L_{b}\left(\Omega_{t}\right)$ : the space of all bounded elements in $L^{0}\left(\Omega_{t}\right)$. Let $\mathbb{L}_{G}^{p}(\Omega)$ be the closure of $\mathscr{H}$ with respect to the norm

$$
\|X\|_{p}=\widehat{\mathbb{E}}\left[|X|^{p}\right]^{1 / p}
$$

with $p \in[1, \infty)$. Clearly, the space $\mathbb{L}_{G}^{p}(\Omega)$ is a Banach space and the space $C_{b}(\Omega)$ of bounded continuous functions on $\Omega$ is a subset of $\mathbb{L}_{G}^{1}(\Omega)$, and, moreover, for the sublinear expectation space $\left(\Omega, \mathbb{L}_{G}^{p}(\Omega), \widehat{\mathbb{E}}\right)$, there exists a weakly compact family $\mathscr{P}$ of probability measures on $(\Omega, \mathscr{B}(\Omega))$ such that

$$
\widehat{\mathbb{E}}=\sup _{P \in \mathscr{P}} E_{P} .
$$

So we can introduce the Choquet capacity $\widehat{C}$ by taking

$$
\widehat{C}(A)=\sup _{P \in \mathscr{P}} P(A), \quad A \in \mathscr{B}(\Omega) .
$$

Definition 4. A set $A \subset \Omega$ is called polar if $\widehat{C}(A)=0$. A property is said to hold "quasi-surely" (q.s.) if it holds outside a polar set. 
By using the above family of probability measures $P$, one can characterize the space $\mathbb{L}_{G}^{p}(\Omega)$ as

$$
\begin{aligned}
\mathbb{L}_{G}^{p}(\Omega)= & \left\{L^{0}(\Omega) \ni X\right. \text { is continuous, q.s., } \\
& \left.\sup _{P \in \mathscr{P}} E_{P}\left[|X|^{p}\right]<\infty\right\} \\
\equiv & \left\{L^{0}(\Omega) \ni X\right. \text { is continuous, q.s., } \\
& \left.\lim _{n \rightarrow \infty} \sup _{P \in \mathscr{P}} E_{P}\left[|X|^{p} 1_{\{|X|>n\}}\right]=0\right\} .
\end{aligned}
$$

The following three results can be consulted in Denis et al. [4] and Hu and Peng [5].

Lemma 5 (Denis et al. [4] and Hu and Peng [5]). Let $\left\{X_{n}, n=\right.$ $1,2, \ldots\}$ be a monotonically decreasing sequence of nonnegative random variances in $C_{b}(\Omega)$. If $X_{n}$ converges to zero q.s. on $\Omega$, then one has

$$
\lim _{n \rightarrow 0} \widehat{\mathbb{E}}\left[X_{n}\right]=0 .
$$

Moreover, if $X_{n} \uparrow X$ and $\widehat{\mathbb{E}}[X]$ and $\widehat{\mathbb{E}}\left[X_{n}\right]$ are finite for all $n=1,2, \ldots$, one then has

$$
\lim _{n \rightarrow 0} \widehat{\mathbb{E}}\left[X_{n}\right]=\widehat{\mathbb{E}}[X] .
$$

Lemma 6 (Denis et al. [4] and Hu and Peng [5]). Let $1 \leq p<$ $\infty$. Consider the sets $\mathbb{L}_{G}^{p}(\Omega)$ and $\mathbb{L}^{p}=\mathscr{L}^{p} / \mathcal{N}$, where

$$
\begin{gathered}
\mathscr{L}^{p}=\left\{X \in L^{0}(\Omega): \widehat{\mathbb{E}}\left(|X|^{p}\right)=\sup _{P \in \mathscr{P}} E_{P}\left[|X|^{p}\right]<\infty\right\}, \\
\mathscr{N}=\left\{X \in L^{0}(\Omega): X=0 \text { q.s. }\right\} .
\end{gathered}
$$

Then,

(i) $\mathbb{L}^{p}$ is a Banach space with respect to the norm $\|\cdot\|_{p}$;

(ii) $\mathbb{L}_{G}^{p}$ is the completion of $C_{b}(\Omega)$ with respect to the norm $\|\cdot\|_{p}$.

Lemma 7 (Denis et al. [4] and Hu and Peng [5]). For a given $p \in(0,+\infty]$, if the sequence $\mathbb{L}^{p} \supset\left\{X_{n}\right\}$ converges to $X$ in $\mathbb{L}^{p}$, then there exists a subsequence $\left\{X_{n_{k}}\right\}$ such that $X_{n_{k}}$ converges to $X$ quasi-surely.

We denote by $\mathbb{L}_{*}^{p}(\Omega)$ the completion of $L_{b}(\Omega)$ with respect to the norm $\|\cdot\|_{p}$.

Definition 8 (G-Brownian motion). A process $B=\left\{B_{t}, t \geq\right.$ $0\} \subset \mathscr{H}$ in a sublinear expectation space $(\Omega, \mathscr{H}, \widehat{\mathbb{E}})$ is called a $G$-Brownian motion if the following properties are satisfied:

(i) $B_{0}=0$;

(ii) for each $t, s \geq 0$, the increment $B_{t+s}-B_{t}$ is $N\left(0,\left[\underline{\sigma}^{2} s, \bar{\sigma}^{2} s\right]\right)$-distributed and is independent from $\left(B_{t_{1}}, \ldots, B_{t_{n}}\right)$, for all $n=0,1,2, \ldots$ and $0 \leq t_{1} \leq t_{2} \leq$ $\cdots \leq t_{n} \leq t$.
The $G$-Brownian motion $B$ has the following properties:

(1) for all $\xi \in \mathbb{L}^{2}\left(\Omega_{t}\right)$, one has $\widehat{\mathbb{E}}\left[\xi\left(B_{T}-B_{t}\right)\right]=0$ with $0 \leq t \leq T$;

(2) for all $\mathscr{B}\left(\Omega_{t}\right)$-measurable real valued, bounded functions $\xi$, one has

$$
\widehat{\mathbb{E}}\left[\xi^{2}\left(B_{T}-B_{t}\right)^{2}\right] \leq \bar{\sigma}^{2}(T-t) \widehat{\mathbb{E}}\left[\xi^{2}\right], \quad 0 \leq t \leq T ;
$$

(3) for all $t \geq 0$, one has $\widehat{\mathbb{E}}\left[B_{t}\right]=\widehat{\mathbb{E}}\left[-B_{t}\right]=0$;

(4) $t \mapsto B_{t}$ is Hölder continuous of order $\delta<1 / 2$, quasisurely.

In Li and Peng [6], a generalized Itô integral and a generalized Itô formula with respect to the $G$-Brownian motion are introduced. For arbitrarily fixed $p \geq 1$ and $T \in \mathbb{R}_{+}$, one denotes by $M_{b}^{p, 0}([0, T])$ the set of step processes as follows:

$$
\eta_{t}(\omega)=\sum_{j=1}^{N} \xi_{j}(\omega) 1_{\left[t_{j-1}, t_{j}\right)}(t), \quad \xi_{j} \in L_{b}\left(\Omega_{t_{j-1}}\right)
$$

with $0=t_{0}<\cdots<t_{N}=T$. For the process of the form (25) one defines the related Bochner integral as follows:

$$
\int_{0}^{T} \eta_{t} d t=\sum_{j=1}^{N} \xi_{j}\left(t_{j}-t_{j-1}\right) .
$$

For every $\eta \in M_{b}^{p, 0}([0, T])$, one sets

$$
\widehat{\mathbb{E}}_{T}(\eta):=\frac{1}{T} \widehat{\mathbb{E}} \int_{0}^{T} \eta_{t} d t
$$

Then $\widehat{\mathbb{E}}_{T}$ forms a sublinear expectation. Moreover, one denotes by $M_{*}^{p}([0, T])$ the completion of $M_{b}^{p, 0}([0, T])$ under the norm

$$
\|\eta\|_{M_{*}^{p}([0, T])}=\left(\widehat{\mathbb{E}}\left[\int_{0}^{T}\left|\eta_{s}\right|^{p} d s\right]\right)^{1 / p} .
$$

Definition 9. For every $\eta \in M_{b}^{p, 0}([0, T])$ of the form (25), one defines the Itô integral of $\eta$ with respect to $G$-Brownian motion $B$ by

$$
I(\eta):=\int_{0}^{T} \eta_{s} d B_{s}=\sum_{j=1}^{N} \xi_{j}\left(B_{t_{j}}-B_{t_{j-1}}\right) .
$$

The mapping $I: M_{b}^{p, 0}([0, T]) \rightarrow \mathbb{L}_{*}^{2}\left(\Omega_{T}\right)$ is a linear continuous mapping and thus can be continuously extended to $I: M_{*}^{2}([0, T]) \rightarrow \mathbb{L}_{*}^{2}\left(\Omega_{T}\right)$, which is called the Itô integral of $\eta \in M_{*}^{2}([0, T])$ with respect to $G$-Brownian motion $B$, and define

$$
\int_{0}^{t} \eta_{s} d B_{s}=\int_{0}^{T} 1_{\{0 \leq s \leq t\}} \eta_{s} d B_{s}
$$


for all $\eta \in M_{*}^{2}([0, T])$ and $t \in[0, T]$. One has

$$
\begin{gathered}
\widehat{\mathbb{E}}\left(\int_{0}^{T} \eta_{s} d B_{s}\right)=0 \\
\widehat{\mathbb{E}}\left[\left(\int_{0}^{T} \eta_{s} d B_{s}\right)^{2}\right] \leq \bar{\sigma}^{2} \widehat{\mathbb{E}}\left[\int_{0}^{T} \eta_{s}^{2} d s\right]
\end{gathered}
$$

for all $\eta \in M_{*}^{2}([0, T])$. Moreover, the process $\left\{\int_{0}^{t} \eta_{s} d B_{s}, t \in\right.$ $[0, T]\}$ is continuous in $t$ quasi-surely and

$$
\int_{0} \eta_{s} d B_{s} \in M_{*}^{2}([0, T])
$$

for all $\eta \in M_{*}^{2}([0, T])$.

Definition 10 (quadratic variation). Let $\pi_{t}^{N}=\left\{0=t_{0}^{N}<t_{1}^{N}<\right.$ $\left.\cdots<t_{N-1}^{N}=t\right\}$ be a partition of $[0, t]$ for $t>0$, such that $\mu\left(\pi_{t}^{N}\right):=\max _{j}\left\{t_{j}-t_{j-1}\right\} \rightarrow 0$ as $N \rightarrow \infty$. The quadratic variation of $G$-Brownian motion $B$ is defined by

$$
\langle B\rangle_{t}=\lim _{\mu\left(\pi_{t}^{N}\right) \rightarrow 0} \sum_{k=0}^{N-1}\left(B_{t_{k+1}^{N}}-B_{t_{k}^{N}}\right)^{2}=B_{t}^{2}-2 \int_{0}^{t} B_{s} d B_{s}
$$

in $\mathbb{L}_{G}^{2}(\Omega)$.

The function $t \mapsto\langle B\rangle_{t}$ is continuous and increasing outside a polar set. One can define the integral

$$
\int_{0}^{T} \eta_{t} d\langle B\rangle_{t}:=\sum_{j=1}^{N} \xi_{j}\left(\langle B\rangle_{t_{j}}-\langle B\rangle_{t_{j-1}}\right)
$$

as a map from $M_{b}^{1,0}([0, T])$ into $\mathbb{L}_{*}^{1}\left(\Omega_{T}\right)$, and the map is linear and continuous, and it can be extended continuously to $M_{*}^{1}([0, T])$.

Definition 11. A process $\left(M_{t}\right)_{t \geqslant 0}$ is called a $G$-martingale if, for each $t \in[0, \infty), M_{t} \in \mathbb{L}_{G}^{1}\left(\mathscr{F}_{t}\right)$ and, for each $s \in[0, t]$, one has

$$
\widehat{\mathbb{E}}\left[M_{t} \mid \mathscr{F}_{s}\right]=M_{s} .
$$

If both $\left(M_{t}\right)_{t \geqslant 0}$ and $\left(-M_{t}\right)_{t \geqslant 0}$ are $G$-martingales, $\left(M_{t}\right)_{t \geqslant 0}$ is called a $G$-symmetric martingale.

One can easily prove that the process

$$
M_{t}:=\widehat{\mathbb{E}}\left[X \mid \mathscr{F}_{t}\right], \quad t \geq 0,
$$

is a $G$-martingale for each $X \in \mathbb{L}_{G}^{1}(\mathscr{F})$, and, moreover, the process

$$
\begin{aligned}
M_{t}:= & M_{0}+\int_{0}^{t} \varphi_{u} d B_{u}+\int_{0}^{t} \eta d\langle B\rangle_{u} \\
& -\int_{0}^{t} 2 G\left(\eta_{u}\right) d u, \quad \forall t \in[0, T]
\end{aligned}
$$

also is a $G$-martingale for all $M_{0} \in \mathbb{R}, \varphi \in M_{b}^{2}[0, T], \eta \in$ $M_{b}^{1}[0, T]$.

\section{Proof of Theorem 1}

Theorem 12. If there exists an $\epsilon>0$ such that

$$
\widehat{\mathbb{E}}\left[\exp \left\{\left(\frac{1}{2}+\epsilon_{0}\right) \int_{0}^{T} H(s, \omega) d B_{s}\right\}\right]<\infty,
$$

then

$$
\mathscr{E}\left(B_{t}\right):=\exp \left\{\int_{0}^{t} H(s, \omega) d B_{s}-\frac{1}{2} \int_{0}^{t} H^{2}(s, \omega) d\langle B\rangle_{s}\right\}
$$

is a symmetric martingale under $\widehat{\mathbb{E}}$, and, for all $t \in[0, T]$, $\mathscr{E}\left(B_{t}\right) \in \mathbb{L}_{G}^{1}\left(\mathscr{F}_{t}\right)$.

Proof. Since $B_{t}$ is a mean-square integrable martingale under each $P_{v}$ and

$$
\begin{aligned}
E_{P_{v}} & {\left[\exp \left\{\frac{1}{2} \int_{0}^{T} H(s, \omega) d B_{s}\right\}\right] } \\
& \leqslant E_{P_{\nu}}^{1 /\left(1+2 \epsilon_{0}\right)}\left[\exp \left\{\left(\frac{1}{2}+\epsilon_{0}\right) \int_{0}^{T} H(s, \omega) d B_{s}\right\}\right] \\
& \leqslant \widehat{\mathbb{E}}^{1 /\left(1+2 \epsilon_{0}\right)}\left[\exp \left\{\left(\frac{1}{2}+\epsilon_{0}\right) \int_{0}^{T} H(s, \omega) d B_{s}\right\}\right]<\infty,
\end{aligned}
$$

it follows from Kazamaki's condition that $\mathscr{E}\left(B_{t}\right)$ is a martingale under each $P_{v}$, and $E_{P_{v}}\left[\mathscr{E}\left(B_{t}\right)\right]=1$. Thus, $\widehat{\mathbb{E}}\left[\mathscr{E}\left(B_{t}\right)\right]=1$, and $\mathscr{E}\left(B_{t}\right)$ is symmetric.

We now claim that $\mathscr{E}\left(B_{t}\right) \in \mathbb{L}_{G}^{1}\left(\mathscr{F}_{t}\right)$. By Lemma 5, it suffices to prove

$$
\lim _{n \rightarrow \infty} \widehat{\mathbb{E}}\left[\mathscr{E}\left(B_{t}\right) I_{\left\{\mathscr{E}\left(B_{t}\right)>n\right\}}\right]=0 .
$$

Now,

$$
\begin{array}{r}
\widehat{\mathbb{E}}\left[\mathscr{E}^{1+k}\left(B_{t}\right)\right] \\
=\widehat{\mathbb{E}}\left[\exp \left\{(1+k) \int_{0}^{t} H(s, \omega) d B_{s}-\frac{1+k}{2} \int_{0}^{t} H^{2}(s, \omega) d\langle B\rangle_{s}\right\}\right] \\
=\widehat{\mathbb{E}}\left[\exp \left\{\frac{\sqrt{1+k}}{\sqrt{1+s}} \int_{0}^{t} H(s, \omega) d B_{s}-\frac{1+k}{2} \int_{0}^{t} H^{2}(s, \omega) d\langle B\rangle_{s}\right\}\right. \\
\left.\times \exp \left\{\left((1+k)-\frac{\sqrt{1+k}}{\sqrt{1+s}}\right) \int_{0}^{t} H(s, \omega) d B_{s}\right\}\right] \\
\leqslant \widehat{\mathbb{E}}^{s /(1+s)}\left[\operatorname { e x p } \left\{\sqrt{(1+k)(1+s)} \int_{0}^{t} H(s, \omega) d B_{s}\right.\right. \\
\left.\left.\quad-\frac{(1+k)(1+s)}{2} \int_{0}^{t} H^{2}(s, \omega) d\langle B\rangle_{s}\right\}\right] \\
\times \widehat{\mathbb{E}}^{s /(1+s)}\left[\exp \left\{\left((1+k)-\frac{\sqrt{1+k}}{\sqrt{1+s}}\right) \frac{1+s}{s} \int_{0}^{t} H(s, \omega) d B_{s}\right\}\right] \\
=\widehat{\mathbb{E}}^{s /(1+s)}\left[\exp \left\{\left((1+k)-\frac{\sqrt{1+k}}{\sqrt{1+s}}\right) \frac{1+s}{s} \int_{0}^{t} H(s, \omega) d B_{s}\right\}\right],
\end{array}
$$


where $k$ and $s$ are small positive numbers. Without loss of generality, let $k=\theta s$. Then we have

$$
\begin{aligned}
& \lim _{s \rightarrow 0}\left((1+k)-\frac{\sqrt{1+k}}{\sqrt{1+s}}\right) \frac{1+s}{s} \\
& =\lim _{s \rightarrow 0} \frac{\sqrt{(1+\theta s)(1+s)}(\sqrt{(1+\theta s)(1+s)}-1)}{s} \\
& =\frac{1}{2}(1+\theta) .
\end{aligned}
$$

So we can choose $\theta$ and $s$ small enough such that

$$
\left((1+k)-\frac{\sqrt{1+k}}{\sqrt{1+s}}\right) \frac{1+s}{s}<\frac{1}{2}+\varepsilon_{0} .
$$

Then, $\widehat{\mathbb{E}}\left[\mathscr{E}^{1+k}\left(B_{t}\right)\right]<\infty$ and

$$
\widehat{C}\left[\mathscr{E}\left(B_{t}\right)>n\right] \leqslant \frac{\widehat{\mathbb{E}}\left[\mathscr{E}\left(B_{t}\right)\right]}{n} \longrightarrow 0,
$$

so that

$$
\begin{aligned}
& \lim _{n \rightarrow \infty} E_{G}\left[\mathscr{E}\left(B_{t}\right) I_{\left\{\mathscr{E}\left(B_{t}\right)>n\right\}}\right] \\
& \quad \leqslant \widehat{\mathbb{E}}^{1 /(1+k)}\left[\mathscr{E}^{1+k}\left(B_{t}\right)\right] \widehat{C}^{k /(1+k)}\left[\mathscr{E}\left(B_{t}\right)>n\right]=0 .
\end{aligned}
$$

Since $\mathscr{E}\left(B_{t}\right) \in L_{G}^{1}\left(\mathscr{F}_{t}\right)$, and $\mathscr{E}\left(B_{t}\right)$ is a martingale under each $P_{v}$, we have

$$
\widehat{\mathbb{E}}\left[\mathscr{E}\left(B_{T}\right) \mid \mathscr{F}_{t}\right]=E_{P_{\nu}}\left[\mathscr{E}\left(B_{T}\right) \mid \mathscr{F}_{t}\right]=\mathscr{E}\left(B_{t}\right), \quad P_{v} \text {. a.s. }
$$

Then,

$$
\begin{aligned}
\widehat{\mathbb{E}} & {\left[\left|\widehat{\mathbb{E}}\left[\mathscr{E}\left(B_{T}\right) \mid \mathscr{F}_{t}\right]-\mathscr{E}\left(B_{t}\right)\right|\right] } \\
& =\sup _{P_{\nu} \in \mathscr{P}} E_{P_{\nu}}\left[\left|\widehat{\mathbb{E}}\left[\mathscr{E}\left(B_{T}\right) \mid \mathscr{F}_{t}\right]-\mathscr{E}\left(B_{t}\right)\right|\right] \\
& =\sup _{P_{\nu} \in \mathscr{P}} E_{P_{\nu}}\left[\left|\mathscr{E}\left(B_{t}\right)-\mathscr{E}\left(B_{t}\right)\right|\right]=0,
\end{aligned}
$$

which means that $\mathscr{E}\left(B_{t}\right)$ is a symmetric martingale.

As a corollary, we can obtain the following criterion, because

$$
\widehat{\mathbb{E}}\left\{\exp \left[\left(\frac{1}{2}+\varepsilon_{0}\right) M_{\infty}\right]\right\} \leqslant \widehat{\mathbb{E}}\left\{\exp \left[\left(\frac{1}{2}+\varepsilon_{0}\right)\langle M\rangle_{\infty}\right]\right\}^{1 / 2} .
$$

Corollary 13. If there exists an $\varepsilon>0$ such that

$$
\widehat{\mathbb{E}}\left[\exp \left\{\left(\frac{1}{2}+\varepsilon_{0}\right) \int_{0}^{T} H^{2}(s, \omega) d\langle B\rangle_{s}\right\}\right]<\infty,
$$

then $\mathscr{E}\left(B_{t}\right)$ is a symmetric martingale under $E_{G}$, and, for all $t \in[0, T], \mathscr{E}\left(B_{t}\right) \in L_{G}^{1}\left(\mathscr{F}_{t}\right)$.
We will close this section with an example which tells a distinct difference between the above two criteria. Let $M_{t}=$ $\int_{0}^{t} H(s, \omega) d B_{s}$.

Theorem 14. If $\|M\|_{\infty}<\pi / 2$, then

$$
\widehat{\mathbb{E}}\left[\exp \left\{\left(\frac{1}{2}+\varepsilon_{0}\right) \int_{0}^{T} H^{2}(s, \omega) d\langle B\rangle_{s}\right\}\right]<\infty .
$$

However, there exists a G-martingale $M$ such that $\|M\|_{\infty}=$ $\pi / 2$ and

$$
\widehat{\mathbb{E}}\left[\exp \left\{\left(\frac{1}{2}+\varepsilon_{0}\right) \int_{0}^{T} H^{2}(s, \omega) d\langle B\rangle_{s}\right\}\right]=\infty .
$$

To show this, one needs the next lemma.

Lemma 15 (see [7]). Let $(M)_{t \geqslant 0}$ is a mean-square integrable martingale, $a, b>0$ and $\tau=\inf \left\{t: M_{t} \notin(-a, b)\right\}$. Then one has

$$
\begin{aligned}
E[ & {\left[\exp \left(\frac{1}{2} \theta^{2}\langle M\rangle_{\tau}\right)\right] } \\
& =\frac{\cos (((a-b) / 2) \theta)}{\cos (((a+b) / 2) \theta)} \quad\left(0 \leqslant \theta<\frac{\pi}{a+b}\right) .
\end{aligned}
$$

Proof of Theorem 14. Firstly, let us consider the stopping time $\tau=\inf \left\{t: B_{t} \notin(-d, d)\right\}$, where $d \leqslant(\pi / 2)$, and consider the process $M=B^{\tau}$. From [8], we know that $(M)_{t \geqslant 0}$ is a $G$-symmetric martingale, and, under each probability measurement $P \in \mathscr{P},(M)_{t \geqslant 0}$ is a mean-square integrable martingale. Then, it follows from the lemma that

$$
\begin{aligned}
E_{P}\left[\exp \left(\frac{1}{2}\langle M\rangle_{\infty}\right)\right] & =E_{P}\left[\exp \left(\frac{1}{2}\langle B\rangle_{\tau}\right)\right] \\
& = \begin{cases}\frac{1}{\cos d}<\infty, & d<\frac{\pi}{2} \\
\lim _{\theta \rightarrow 1} \frac{1}{\cos ((\pi / 2) \theta)}=\infty, & d=\frac{\pi}{2}\end{cases}
\end{aligned}
$$

that is, we use the relationship $\widehat{\mathbb{E}}[\cdot]=\sup _{P \in \mathscr{P}} E_{P}[\cdot]$ and complete the proof.

\section{Acknowledgments}

The project is sponsored by NSFC (11171062) and the Innovation Program of Shanghai Municipal Education Commission (12ZZ063).

\section{References}

[1] S. Peng, "Nonlinear expectations andstochastic calculus under uncertainty, preprint," Probability. In press.

[2] S. Peng, G-Expectation, G-Brownian Motion and Related Stochastic Calculus of Itô Type, Springer, Berlin, Germany, 2007, Stochastic Analysis and Applications the Abel Symposium.

[3] J. Xu, H. Shang, and B. Zhang, "A Girsanov type theorem under G-framework," Stochastic Analysis and Applications, vol. 29, no. 3, pp. 386-406, 2011. 
[4] L. Denis, M. Hu, and S. Peng, "Function spaces and capacity related to a sublinear expectation: application to $G$ motion paths," Potential Analysis, vol. 34, no. 2, pp. 139-161, 2011.

[5] M.-S. Hu and S.-G. Peng, "On representation theorem of G-expectations and paths of G-Brownian motion," Acta Mathematicae Applicatae Sinica, vol. 25, no. 3, pp. 539-546, 2009.

[6] X. Li and S. Peng, "Stopping times and related Itô's calculus with G Brownian motion," Stochastic Processes and their Applications, vol. 121, no. 7, pp. 1492-1508, 2011.

[7] N. Kazamaki, Continuous Exponential Martingales and BMO, vol. 1579, Springer, Berlin, Germany, 1994, Lecture Notes in Mathematics.

[8] Y. Song, "Properties of hitting times for G-martingales and their applications," Stochastic Processes and Their Applications, vol. 121, no. 8, pp. 1770-1784, 2011. 


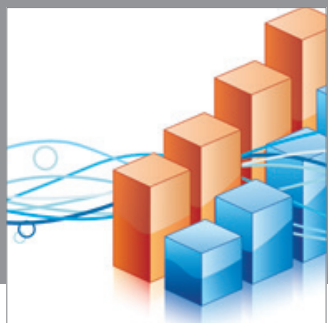

Advances in

Operations Research

mansans

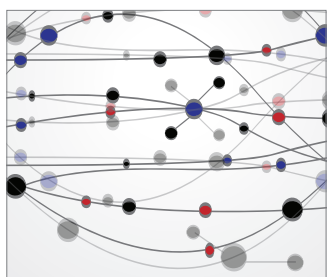

The Scientific World Journal
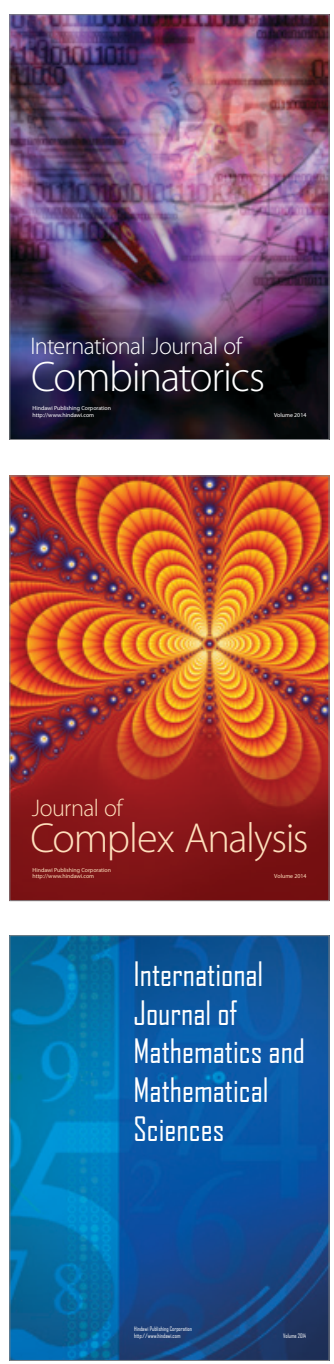
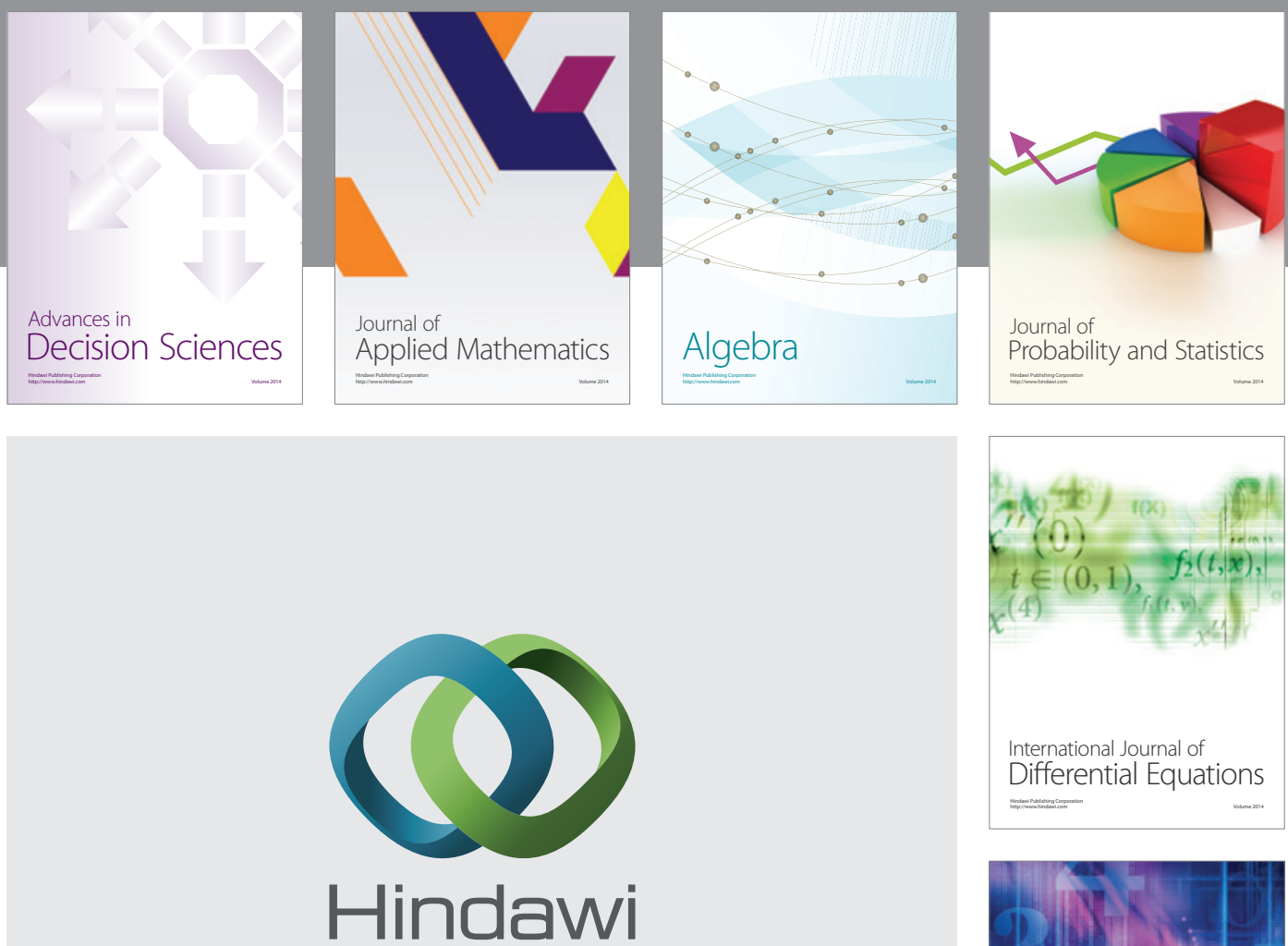

Submit your manuscripts at http://www.hindawi.com
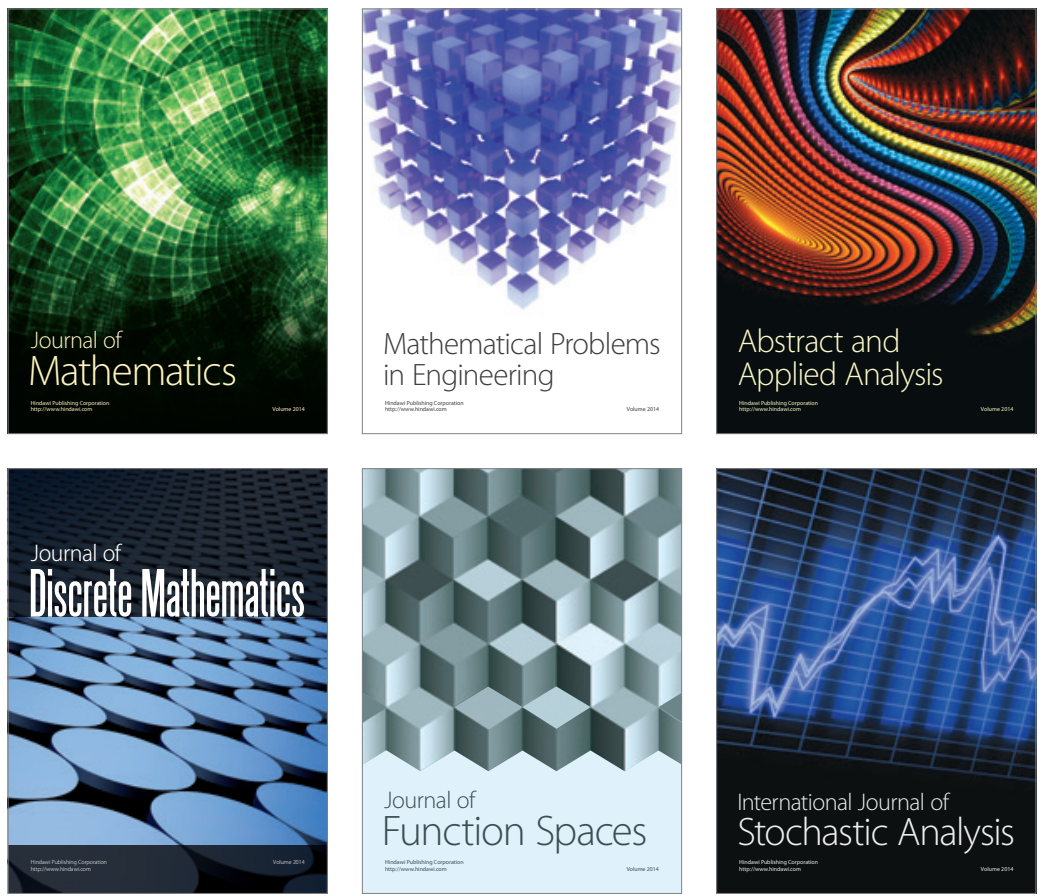

Journal of

Function Spaces

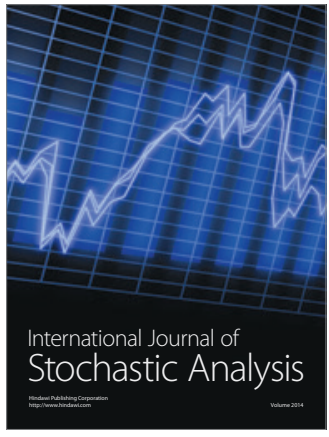

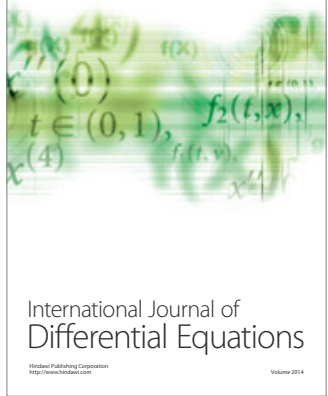
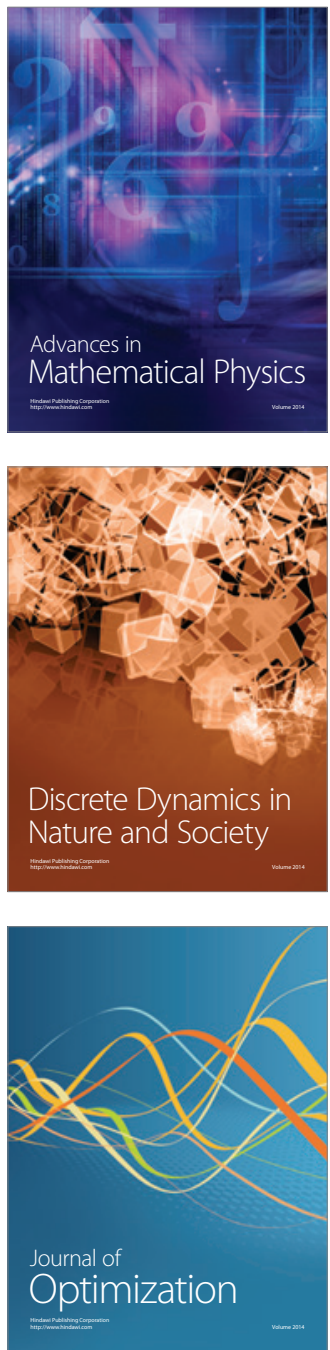